\title{
Tracking Individual Kinesin Motors in Living Cells Using Single Quantum-Dot Imaging
}

2006

Vol. 6, No. 7

1491-1495

\author{
Sébastien Courty, ${ }^{\dagger}$ Camilla Luccardini, ${ }^{\dagger}$ Yohanns Bellaiche, ${ }^{\ddagger}$ \\ Giovanni Cappello, ${ }^{*} \S$ and Maxime Dahan ${ }^{*, \dagger}$

\begin{abstract}
Laboratoire Kastler Brossel, CNRS UMR 8552, Ecole normale supérieure, Université
Pierre et Marie Curie-Paris 6, 24 rue Lhomond 75005 Paris, France, Institut Curie,

CNRS UMR 144, 26 rue d'Ulm 75005 Paris, France, and Institut Curie,

CNRS UMR 168, 26 rue d'Ulm 75005 Paris, France
\end{abstract}

Received April 25, 2006; Revised Manuscript Received June 7, 2006

\begin{abstract}
We report a simple method using semiconductor quantum dots (QDs) to track the motion of intracellular proteins with a high sensitivity. We characterized the in vivo motion of individual QD-tagged kinesin motors in living HeLa cells. Single-molecule measurements provided important parameters of the motor, such as its velocity and processivity, as well as an estimate of the force necessary to carry a QD. Our measurements demonstrate the importance of single-molecule experiments in the investigation of intracellular transport as well as the potential of single quantum-dot imaging for the study of important processes such as cellular trafficking, cell polarization, and division.
\end{abstract}

The ability to observe the motion of individual biomolecules provides a powerful tool for investigating the molecular dynamics within living cells and for obtaining information that remains elusive for conventional imaging methods. Single-molecule imaging in living cells has become possible recently ${ }^{1-4}$ but has remained limited, with the exception of a few systems, ${ }^{5-8}$ to the dynamics of molecules diffusing in the cell membrane. In comparison to more established optical methods using GFP and its variants, it allows for a direct determination of molecular motions without averaging over a large number of molecules and therefore permits the identification of distinct molecular subpopulations as well as the analysis of the kinetic parameters controlling their dynamics. In this context, new fluorescent inorganic probes such as semiconductor quantum dots (QDs) provide great prospects for ultrasensitive imaging. ${ }^{4,9}$ Their photostability and high extinction coefficient, compared with organic fluorophores, enable long-term observations at the single nanoparticule level in an optically noisy environment such as live cells. QDs have, for instance, allowed the diffusion dynamics of glycine receptors in the membrane of live neurons to be studied with great accuracy. ${ }^{4}$ Here we went beyond experiments with membrane molecules and showed that QDs could be used successfully to accomplish a more

\footnotetext{
* Corresponding authors. E-mail: giovanni.cappello@curie.fr; maxime.dahan@1kb.ens.fr.

† Laboratoire Kastler Brossel, CNRS UMR 8552.

$\div$ Institut Curie, CNRS UMR 144.

$\S$ Institut Curie, CNRS UMR 168.
}

challenging task: observing the intracellular motion of individual QD-tagged proteins in a living cell.

Kinesin molecules are linear molecular motors, which play a central role in many biological processes such as motility, cell division, or intracellular transport. ${ }^{10-12}$ The study of kinesins has benefited greatly, at both an experimental and conceptual level, from the development of single-molecule in vitro experiments. In vitro assays ${ }^{13,14}$ and micromanipulation measurements ${ }^{15}$ have been used to investigate the biochemical and mechanical cycles of kinesin motors, as well as the connection between the two. ${ }^{10}$ How the in vitro findings relate to the properties and functional role of individual motors in living cells, however, remains unexplored, largely because of a lack of experimental methods to detect single cytoplasmic proteins in vivo. In general, the characteristics of their in vivo motions have been inferred from measurements of the transport of small organelles. ${ }^{10}$ Because organelles are often carried by multiple motors, these measurements do not, however, probe the behavior of a single kinesin and provide only an indirect description of its properties.

To investigate the behavior of individual motors in live cells, we used biotinilated conventional Drosophilae kinesins (HA-kinesin KinBio401) ${ }^{16}$ coupled to QD-streptavidin conjugates (QD-SAVs, Quantum Dot Corporation). After expression, purification, and flash freezing (see the Supporting Information), the fraction of active kinesin dimers, estimated with an ATPase activity assay, ${ }^{17}$ was on the order of $1-4 \%$ 

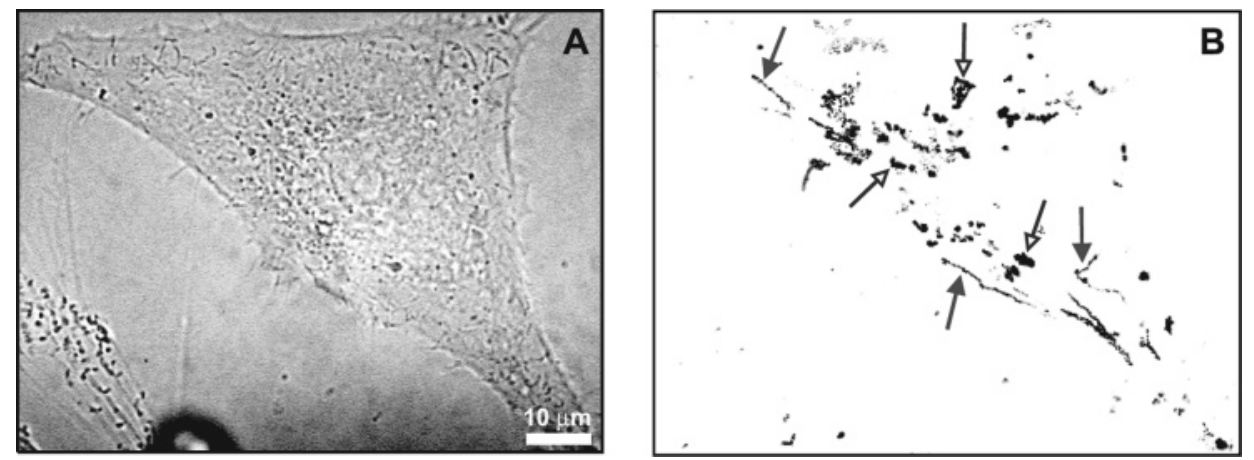

Figure 1. Single QD-kinesin motions in a living cell. (A) Bright-field image of a HeLa cell plated onto a glass coverslip (image size: 89 $\times 67 \mu \mathrm{m}^{2}$ ). (B) Image obtained by superimposing the 600 consecutives frames in the image sequence (time resolution: $230 \mathrm{~ms}$ ). The linear trajectories are indicative of directed motions of individual motors. Examples are marked by the full arrows. The trajectories of diffusing QD-Ks (marked by empty arrowheads) have a random shape in the superimposed image.

of the total number of motors. To prepare complexes with at most one active protein, we mixed QD-SAVs and the kinesins (either active or inactive) with a relative stoechiometry 1:1 or 1:10 (QD/kinesin). The kinesins were always used in the first 3-4 $\mathrm{h}$ after unfreezing. QDs emitting in the red at $655 \mathrm{~nm}$ were used to overcome the cell autofluorescence and achieve a higher signal-to-noise ratio.

We first used conventional in vitro bead assays to determine the velocity and processivity of our QD-kinesin (QD-K) constructs (prepared with either stoechiometry) and to ensure that single motors were observed. In vitro motility experiments were performed at $37^{\circ} \mathrm{C}$ by tracking the motions of single QD-Ks along MTs at a saturating ATP concentration $(1 \mathrm{mM})$, similar to the concentration in the cytosol. The mean velocity and processivity times were equal to $0.60 \pm$ $0.01 \mu \mathrm{m} / \mathrm{s}$ and $1.73 \pm 0.09 \mathrm{~s}(n=97)$, respectively (see the Supporting Information, Figure S1), in agreement with previous values of in vitro assays for individual conventional kinesin motors. ${ }^{18,19}$

QD-Ks were internalized in the cytoplasm of cultured mammalian HeLa cells (Figure 1) with a cell-loading technique based on the osmotic lysis of pinocytic vesicles. ${ }^{20}$ This loading method led to no visible alteration in the cell morphology and was both simpler and more reproducible than microinjection. As shown below, the internalized constructs were located in the cytosol, therefore overcoming the limitations of other methods in which the nanoparticles remain trapped in the endocytotic pathway. ${ }^{21} \mathrm{QD}-\mathrm{Ks}$ were detected with a standard epifluorescence microscope in a temperature-controlled environment at $37^{\circ} \mathrm{C}$, and sequences of fluorescence images were recorded with a time resolution between $75 \mathrm{~ms}$ and $230 \mathrm{~ms}$.

The images first revealed that QD-Ks were distributed homogeneously in the cytoplasm of the cell and did not enter into the nucleus. Because of the spreading of the HeLa cells on the microscope coverslip, the QD motion remained twodimensional and the trajectories rarely exited the focal plane. Using fluorescence intermittency (i.e., the alternation of periods of bright and dark fluorescence emission) as a signature for a single QD, ${ }^{22}$ only "blinking" trajectories were selected for analysis. ${ }^{23}$ To further ensure that blinking QDs observed in the live cell correspond to single QDs, we have mixed two samples of QDs (emitting at 605 and $655 \mathrm{~nm}$ ) before internalization in HeLa cells. We found that the blinking fluorescence spots at 605 and $655 \mathrm{~nm}$ were not colocalized within the cells, indicating that QDs were released in the cytosol as single nanoparticles and not as small aggregates.

We analyzed the trajectories of QD-Ks (prepared with a coupling stoichiometry $1: 10$ ) by computing the mean-square displacement function (MSD). Two main populations could be qualitatively identified: $\sim 13 \%$ of the QDs moved along a linear trajectory while the others displayed a more random behavior. For the latter population, the MSD was linear, evidence for Brownian motion, with a mean diffusion coefficient $D \pm \mathrm{SEM}=0.031 \pm 0.002 \mu \mathrm{m}^{2} / \mathrm{s}(n=75)$. This value is consistent with previous measurements of the diffusion of biomolecules in the cytosol, ${ }^{24}$ when accounting for the hydrodynamic radius $R_{\mathrm{h}} \approx 15 \mathrm{~nm}$ (data not shown) of QD-SAVs. For QD-Ks with a linear trajectory, the MSD had a quadratic time-dependence, indicative of a directed motion. We further quantified the QD-K motion by analyzing the consecutive positions $x(t)$ and $y(t)$ for around 80 trajectories recorded in 11 different cells. In general, the trajectories revealed one or several phases of rapid directed displacement separated by periods of nondirectional motion (Figure 2).

We first directed our analysis on the phases of directed motion. By measuring the velocity of the motors during these phases as well as their duration (i.e., the motor temporal processivity), we were able to characterize for the first time the dynamics of individual kinesin motors in vivo. By analyzing $n=385$ events, we found that the velocity distribution had an average value $v=0.57 \pm 0.02 \mu \mathrm{m} / \mathrm{s}$ (Figure $3 \mathrm{a}$ ) and the mean processivity of the motor was 1.73 $\pm 0.06 \mathrm{~s}$ (Figure $3 \mathrm{~b}$ ). To further support the idea that the directed motions resulted from individual active kinesins, we compared the values of the velocity and the processivity for the two coupling stoichiometries 10:1 and 1:1 and found similar results (see the Supporting Information, Figure S2). The main difference between the two conditions was the fraction of directed events among the trajectories in the cell, which dropped to $\sim 4 \%$ when using QD-Ks prepared with a 1:1 molar ratio. This change is qualitatively consistent with 

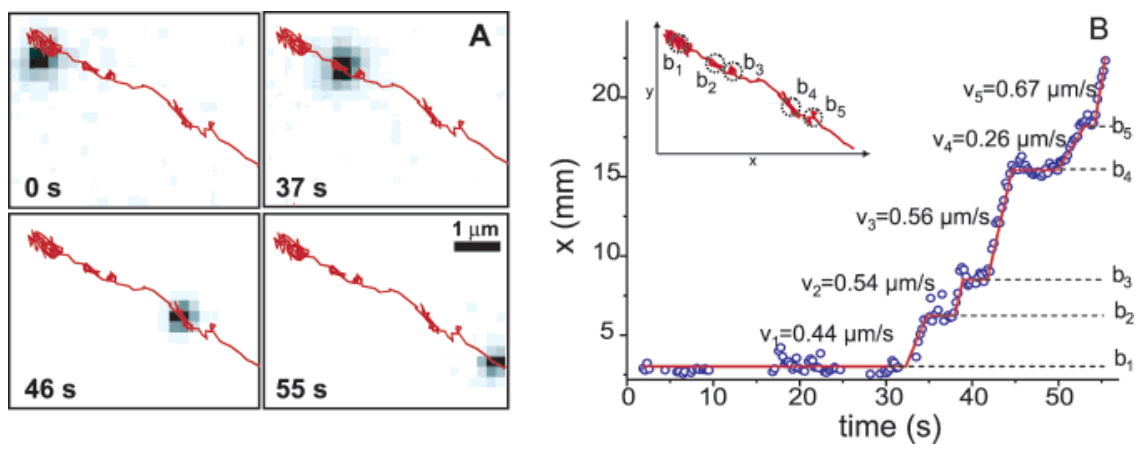

Figure 2. (A) Tracking of a single QD-K in a part of the cell. (B) Inset: trajectory of the QD-K shown in A. The position along $x$ vs time highlights the succession of directed (with corresponding velocities $v_{i}$ ) and diffusive motions (marked by $b_{i}$ ).
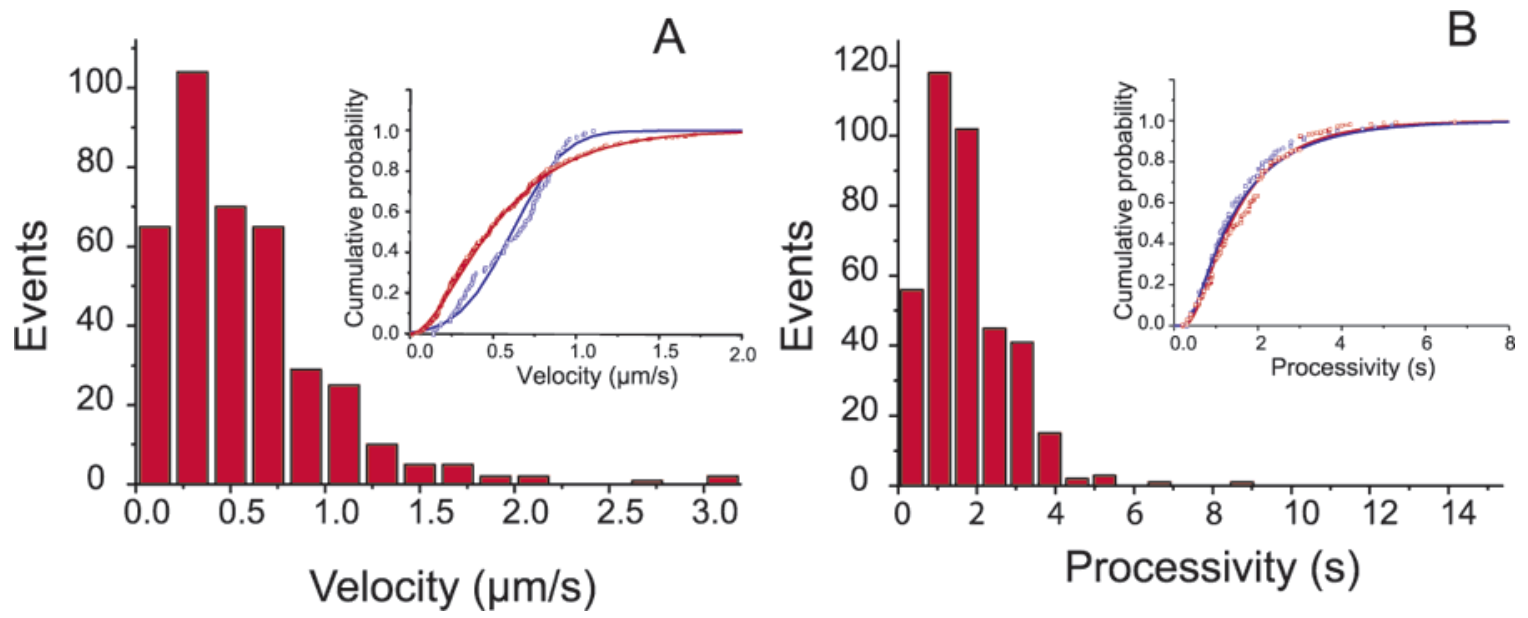

Figure 3. Velocity (A) and processivity (B) distributions of QD-Ks measured in vivo. Insets: cumulative distributions for the in vivo (red) and in vitro (blue) data. The plain lines are Gaussian fits used as guide to the eye.

the fraction of active kinesins and the number of free avidin moieties per QD ( $\sim 5-10$, estimated by the commercial provider).

Additional experiments were carried out to ensure that the directed motions observed in the living cells were specifically due to the motility of kinesin molecules. To this aim, we analyzed the movement after internalization of unconjugated QD-SAVs, QDs solely functionalized with $\mathrm{NH}_{2}$ groups, and QDs coupled to denaturated kinesins (kinesins left $12 \mathrm{~h}$ at 4 ${ }^{\circ} \mathrm{C}$ after unfreezing and for which no movements could be observed in vitro). None of them (out of $\sim 330$ tracks) had a directed motion. They all displayed a characteristic Brownian diffusion with a coefficient $D=0.033 \pm 0.002$ $\mu \mathrm{m}^{2} / \mathrm{s}(n=45)$ (see the Supporting Information, Figure S3), very similar to those measured for unconjugated QDs microinjected in the cytoplasm $\left(D=0.035 \pm 0.005 \mu \mathrm{m}^{2} / \mathrm{s}\right.$, $n=26$ ). This observation implies that the directed trajectories are not caused by QD-Ks contained within vesicles being transported by molecular motors.

The distributions of in vivo and in vitro velocity and processivity are remarkably similar, as shown on the cumulative probability curves (insets in Figure 3). This observation indicates that the motility of QD-Ks in live cells is not affected by the presence of microtubule-associated proteins (MAPs) and by the traffic of other kinesins or dyneins motors competing for binding sites to microtubules. Our measurements extend to the case of live cells the results of previous in vitro motility assays showing that the mechanochemical properties of individual kinesin motors are insensitive to the degree of crowding on microtubules, either with MTassociated proteins ${ }^{25}$ or with others kinesin motors. ${ }^{26}$ Seitz and Surrey distinguished further the effect of mobile versus immobile obstacles. ${ }^{26}$ In both cases, the run length and the mean velocity remained constant, respectively equal to $1 \mu \mathrm{m}$ and $0.6 \mu \mathrm{m} / \mathrm{s}$ (see figure 3 in ref 25) and very similar to our in vivo results. However, the presence of immobile obstacles induced pauses in the kinesin motion, corresponding to a motor waiting for the obstacle to detach from MT and it increased the mean duration of the run lengths. In our measurements, we did not observe any pause, thus indicating that obstacles, if any, are of the mobile type. Our data also reveal that the motion of a kinesin motor, with a QD acting as a cargo, is not modified because of steric hindrance by other cytosolic components, such as the actin meshwork, which contribute to the crowded environment inside the cell. It should be noted, however, that our experiments were performed with exogenous truncated molecular motors. In the case of endogenous motors, we cannot exclude that cytoplasmic cofactors might regulate the properties of kinesins bound to their cargo, including their velocity and their affinity for MTs.

We also performed measurements to investigate the dependency of the QD-K motions on the dynamics of the cytoskeleton. We first determined the effect of actomyosin- 

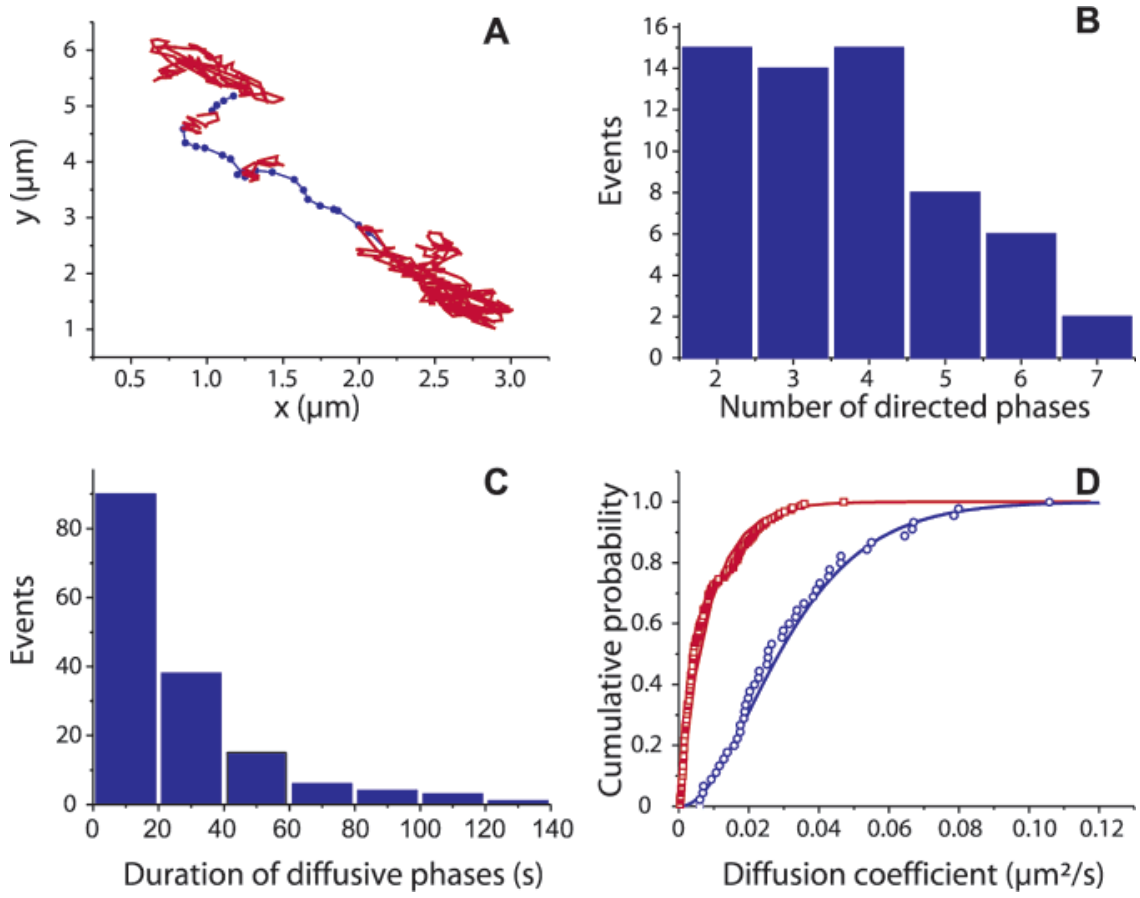

Figure 4. (A) Trajectory of a single QD-K. The plot shows the alternated phases of directed (black dots) and diffusive motions (red line). (B) Number of phases of directed motions, for the 60 trajectories showing a succession of directed and diffusive movements. (C) Distribution of duration of the diffusive phases. (D) Cumulative probability of diffusion coefficient for the diffusive phases (square, red) and for unconjugated QD-SAVs in the cytosol (circle, blue). The cumulative distributions show a significant difference $\left(P<10^{-5}, \mathrm{Kolmogorov}-\right.$ Smirnov test). The plain lines are Gaussian fits used as a guide to the eye.

dependent movement by incubating the HeLa cells with 1 $\mu \mathrm{M}$ of latrunculine $\mathrm{A}$, known to depolymerize the filamentous actin meshwork. The velocity was found to remain unchanged after treatment with the drug (data not shown), demonstrating that the motion of single QD-Ks were independent of the F-actin cytoskeleton.

To disrupt the MT cytoskeleton, we incubated the cells with $10 \mu \mathrm{M}$ of nocodazole, a MT-depolymerizing drug. For 10 different cells, we analyzed the kinesin motions both before and after incubation of the cells with nocodazole. In all cases, the directed motions were suppressed by the drug treatment (see the Supporting Information, Figure S4). We further analyzed the MT polymerization process to ensure that the directed motions were not due to proteins being carried by growing MTs. We quantified the polymerization velocity of MTs in living cells by measuring the motion of EB1, a protein known to present a high affinity for the MT plus-end. ${ }^{27}$ We first tracked the motion of EB1-GFP in transfected Hela Cells (see the Supporting Information, Figure S5) and found velocities centered on $0.165 \pm 0.007$ $\mu \mathrm{m} / \mathrm{s}(n=27)$. This value, in agreement with previous measurements, ${ }^{28}$ is four times slower than the mean velocity obtained for individual kinesins $(\sim 0.6 \mu \mathrm{m} / \mathrm{s})$, showing that the directed motions of individual QD-Ks are not induced by the MT-polymerization. We also performed a similar experiment using purified EB1 conjugated to Cy3 dyes and internalized in HeLa cells with the pynocitic loading reagent. We found a mean velocity equal to $0.14 \pm 0.02 \mu \mathrm{m} / \mathrm{s}(n=$ 7 ), close to the one measured with EB1-GFP, which further validated our approach for injecting molecular constructs into living cells.
In $75 \%$ of the trajectories (60 out of 80 ), we observed processes in which individual kinesins alternated between phases of directed motions along MTs and phases of nondirectional movement (Figures 1c and 3a). Up to seven distinct phases of directed movements could be detected for a single QD-K in a given trajectory ${ }^{29}$ (Figure $3 b$ ). We almost never observed a change in the direction of motion between the consecutive phases. The nondirectional movements corresponded to Brownian diffusion with a mean coefficient $D=0.0083 \pm 0.0007 \mu \mathrm{m}^{2} \cdot \mathrm{s}^{-1}(n=157)$ and had on average a duration $25 \pm 2 \mathrm{~s}$ (Figure 3c). The value of $D$ is four times lower than that measured for unconjugated QDs-SAV diffusing in the cytosol (Figure 3d). Assuming a pure hydrodynamic regime for the friction, we deduced an estimate of the viscosity $\eta=k T /\left(6 \pi R_{\mathrm{h}} D\right) \approx 1.62 \mathrm{~Pa} \cdot \mathrm{s}$ in the local environment of MTs. The force necessary to carry a QD (with a diameter $d$ of $30 \mathrm{~nm}$ ) is then $F=3 \pi \eta \mathrm{d} v \approx 0.6$ $\mathrm{pN}$, well below the stall force $F_{\mathrm{s}}(\sim 6 \mathrm{pN})$.

As a result of this succession of directed and diffusive motions, corresponding to a single motor detaching and reattaching to MTs, the total distance covered by the kinesin was much larger than the typical distance $(\sim 1 \mu \mathrm{m})$ expected from the velocity and processivity of one single motor. The effective distance, defined as the distance between the beginning of the first directed motion and the end of the last one, was about $5.6 \mu \mathrm{m}$, covered in $\sim 70 \mathrm{~s}$. Because of the transient unbinding of the motor, the corresponding apparent velocity was much lower $(\sim 0.08 \mu \mathrm{m} / \mathrm{s})$ than the mean velocity determined from the sole periods of directed motions. To our knowledge, this phenomenon of directed/ diffusive phases has not been described previously for the 
transport of small organelles or vesicles that might be kept attached to microtubules because of the presence of multiple motors.

Single QD experiments enable the analysis of the motor properties of individual kinesin molecules in living cells, extending from previous in vitro analyses of these molecules. Investigations of individual motors in live cells are particularly important in light of recent experiments on the movement of fluorescent endosomes. Using highly accurate localization techniques, different groups have measured their velocity distributions and reported the observation of individual steps in the displacement of single endosomes containing a large number of GFPs or QDs. ${ }^{30-32}$ In our case, the fluorescence signal of an individual QD was not sufficient to reach the temporal and spatial resolution necessary to detect these steps. However, experiments on single endosomes do not enable accurate control over the number and nature of the motors per endosome. As a result, it is difficult to relate their velocity distribution to the mechanical properties of the motors carrying the endosome and, for instance, to explain the high speeds that have been measured (up to $12 \mu \mathrm{m} / \mathrm{s}$ for individual peroxisomes ${ }^{30}$ ). In contrast, experiments on single kinesins provide key parameters, such as the motor processivity, which are not accessible with measurements on endosomes and which are essential for the modeling of vesicle transport. ${ }^{33}$

The above results validate the use of QDs for intracellular single-molecule imaging, and the method can, in principle, be used in the investigation of endogenous intracellular molecules, provided that they can be targeted by functionalized QDs inserted in the cytoplasm. Our measurements therefore represent a first step toward experiments in which QDs, with distinct emission colors and bound to specific biomolecules (either in the membrane, the cytoplasm, or the nucleus), are tracked simultaneously and in real time. Depending on cell lines and molecular targets, this will possibly require nanoparticles with smaller hydrodynamic radii. We thus anticipate that fine adjustment of the QD colloidal properties through their surface coating will be critical for the investigation of intracellular processes at the single-particle level.

Acknowledgment. We gratefully acknowledge J.S. Tirnauer (University of Connecticut) and A. Bershadsky (Weizmann Institute) for their generous gift of His-tag EB1 plasmid and GFP-EB1 construct, respectively. We also thank C. Leduc, P. Bassereau, and J. Prost for fruitful discussions, S. Miserey for her help in the EB1 experiments, A. Echard for his help in the ATPase activity measurements, and M. Hoffman and M. Morgan for careful reading of the manuscript. S.C. was supported by a fellowship from the "Fondation pour la Recherche Medicale (FRM)" and C.L. by a postdoctoral fellowship from CNRS.

Supporting Information Available: Experimental details including protein expression and purification, coupling to QD-streptavidin conjugates, cell loading technique, microtubule and actin filament depolymerization, EB1 coupling reaction to CY3 dyes, single QD tracking and data analysis, in vitro motility assays, and HeLa cell culture. Figures S1S5. A movie, M1, showing the motion of a single QD-K along a microtubule in a live cell is also included. This material is available free of charge via the Internet at http:// pubs.acs.org.

\section{References}

(1) Schutz, G. J.; Kada, G.; Pastushenko, V. P.; Schindler, H. EMBO J. 2000, 19, 892-901.

(2) Harms, G. S.; Cognet, L.; Lommerse, P. H.; Blab, G. A.; Kahr, H.; Gamsjager, R.; Spaink, H. P.; Soldatov, N. M.; Romanin, C.; Schmidt, T. Biophys J. 2001, 81, 2639-2646.

(3) Iino, R.; Koyama, I.; Kusumi, A. Biophys J. 2001, 80, 2667-2677.

(4) Dahan, M.; Levi, S.; Luccardini, C.; Rostaing, P.; Riveau, B.; Triller, A. Science 2003, 302, 442-445.

(5) Deich, J.; Judd, E. M.; McAdams, H. H.; Moerner, W. E. Proc. Natl. Acad. Sci. U.S.A. 2004, 101, 15921-15926.

(6) Yang, W.; Gelles, J.; Musser, S. M. Proc. Natl. Acad. Sci. U.S.A. 2004, 101, 12887-12892.

(7) Shav-Tal, Y.; Darzacq, X.; Shenoy, S.; Fusco, D.; Janicki, S. M.; Spector, D. L.; Singer, R. H. Science 2004, 304, 1797-1800.

(8) Seisenberger, G.; Ried, M. U.; Endress, T.; Buning, H.; Hallek M.; Brauchle, C. Science 2001, 294, 1929-1932.

(9) Michalet, X.; Pinaud, F. F.; Bentolila, L. A.; Tsay, J. M.; Doose, S.; Li, J. J.; Sundaresan, G.; Wu, A. M.; Gambhir; S. S.; Weiss, S. Science 2005, 307, 538-544.

(10) Howard, J. Mechanics of Motor Proteins and the Cytoskeleton; Sinauer: Sunderland, MA, 2001.

(11) Schliwa, M. Molecular Motors; Wiley-VCH: New York, 2003.

(12) Vale, R. D. Cell 2003, 112, 467-480.

(13) Gelles, J.; Schnapp, B.; Sheetz, M. Nature 1987, 331, 450-453.

(14) Vale, R. D.; Funatsu, T.; Pierce, D. W.; Romberg, L.; Harada, Y.; Yanagida, T. Nature 1996, 380, 451-453.

(15) Svoboda, K.; Schmidt, C.; Schnapp, B.; Block, S. M. Nature 1993, $365,721-727$.

(16) Surrey, T.; Elowitz, M. B.; Wolf, P. E.; Yang, F.; Nedelec, F.; Shokat, K.; Leibler, S. Proc. Natl. Acad. Sci. U.S.A. 1998, 95, 4293-4298.

(17) Echard, A.; Jollivet, F.; Martinez, O.; Lacapère, J. J.; Rousselet, A.; Janoueix-Lerosey, I.; Goud, B. Science 1998, 279, 580-585.

(18) Cappello, G.; Badoual, M.; Ott, A.; Prost, J.; Busoni, L. Phys Rev. E 2003, 68, 021907

(19) Friedman, D. S.; Vale, R. D. Nat. Cell Biol. 1999, 1, 293-297.

(20) Okada, C. Y.; Rechsteiner, M. Cell 1982, 29, 33-41.

(21) Jaiswal, J. K; Mattoussi, H.; Mauro, J. M.; Simon, S. M. Nat. Biotechnol. 2003, 21, 47-51.

(22) Shimizu, K. T.; Neuhauser, R. G.; Leatherdale, C. A.; Empedocles, S. A.; Woo, W. K.; Bawendi, M. G. Phys. Rev. B 2001, 63, 205316

(23) Bonneau, S.; Cohen, L.; Dahan, M. Proc. IEEE Int. Symp. Biol. Imaging 2004, 664-668.

(24) Verkman, A. S. Trends Biochem. Sci. 2002, 27, 27-33.

(25) Seitz, A.; Kojima, H.; Oiwa, K.; Mandelkow, E. M.; Song, Y. H.; Mandelkow, E. EMBO J. 2002, 21, 4896-4905.

(26) Seitz, A.; Surrey, T. EMBO J. 2006, 25, 267-277.

(27) Tirnauer, J. S.; Grego, S.; Salmon, E. D; Mitchison, T. J. Mol. Biol. Cell. 2002, 13, 3614-26.

(28) Piehl, M.; Cassimeris, L. Mol. Biol. Cell. 2003, 14, 916-925.

(29) We found that the fluctuations of the velocity in the successive periods of directed motions were much less than those of the full distribution (Figures $2 \mathrm{~b}$ and $3 \mathrm{a}$ ). The relative standard deviation in a given trajectory was on average equal to $0.22 \pm 0.02(n=60)$, lower than 0.77 , the relative standard deviation computed for the full distribution. This finding further supports the idea that the width of the velocity distribution results from heterogeneity between the purified kinesins and that the directed motions are due to individual active motors

(30) Kural, C.; Kim, H.; Goshima, G.; Gelfand, V. I.; Selvin, P. R. Science 2005, 308, 1469-1472.

(31) Nan, X.; Sims, P. A.; Chen, P.; Xie, X. S. J. Phys. Chem. B 2005, 109, 24220.

(32) Levi, V.; Serpinskaya, A. S.; Gratton, E.; Gelfand, V. Biophys. J. 2006, 90, 318-327.

(33) Klumpp, S.; Lipowsky, R. Proc. Natl. Acad. Sci. U.S.A. 2005, 102, $17284-17289$

NL060921T 